\title{
From planning sustainable cities to designing resilient urban regions
}

\author{
D. Bogunovich \\ School of Architecture and Landscape Architecture, Unitec New Zealand, \\ Auckland, New Zealand
}

\begin{abstract}
In order to be ready for the harsh environmental challenges of the $21^{\text {st }}$ century, cities need a new planning agenda. Defining this agenda is impossible without first revisiting the core concepts of our work: 'sustainability', 'city' and 'urban planning'. A four-fold shift in emphasis is vital: from an excessive focus on the development density, to the monitoring and taming of urban metabolism; from a single focus on sustainability to one which also includes resilience; from traditional city to urban region; from policy and land-use planning to strategic urban and regional design. In most parts of the world, the battle for the compact city is lost. However, from the perspective of ecological sustainability, perhaps it was the wrong battle anyway. We should now direct our creative energies at deploying ecological design and clean technologies on a mass scale to our unstoppable, sprawling urban regions.
\end{abstract}

Keywords: sustainability, resilience, urban metabolism, urban regions.

\section{Introduction: a shift in emphasis}

Urban sprawl has been the subject of political and professional debates for more than 30 years. However, the question of what should be done about it - if anything at all - has not yet had a clear-cut answer.

For most planners, designers, bureaucrats and politicians this has been the cause of considerable frustration. But they should take consolation in the fact that the messy debate has been fruitful otherwise. It has brought up to the surface two important trends. One of these is a shift in the perception of the problem: What started as concern over a nexus of primarily social or cultural issues (such as alienation and ugliness), over time became more focused towards 
environmental and economic impacts ('the costs of sprawl'). An important consequence of this shift was that, while in the beginning, some commentators dismissed the debate as an exaggerated problem in an essentially affluent and happy society, recently the debate has been seen as a matter of survival of the human species, or at least urban civilization. The stakes have risen.

The second perceptible trend has been a shift in the balance between the two sides in the debate - the critics vs. the defenders of urban sprawl. Having an advantage from the beginning by the sheer fact that they started the debate, the critics of sprawl always were in majority. The opponents were a vociferous minority, particularly during the laissez faire eighties. Their key argument has typically been to highlight advantages of suburban living, if for no other reason than to maintain a balanced view on the issue. The more ideologically driven among them had a deeper case; for them this was all about basic freedom. They insisted that as a matter of principle, people should be free to choose how and where they wanted to live. They also argued that the 'free market' was a far superior method to deliver that freedom of choice than 'bureaucratic' planning. If the aggregate outcome of all these individual decisions was suburban sprawl, then that's what the people wanted and we should leave them alone.

Perhaps this position had some merit in the $20^{\text {th }}$ century and, if nothing else, should be tolerated as an interesting alternative point of view. But ever since the dual threat of global climate change and the shortages (and/or steeply rising prices) of such key commodities as fossil fuel, clean water and food, has knocked at our doors, the position of the apologists of urban sprawl has become untenable.

Is this then the 'end of history' of the debate on urban sprawl? Hardly so. The debate is only changing its dominant concern. Almost imperceptibly, it is turning from a debate about 'urban growth management' into a debate about 'urban un/sustainability'. As this historic shift takes place, we need to acknowledge that important assumptions on both sides of the fence in the urban sprawl debate - while well intended and mostly well founded some 30 or 20 years ago - have now reached their expiry date. Due to a host of technological and cultural changes that have affected us around the turn of the century, and due to the almost daily worsening forecasts on the condition of this planet's biosphere, certain priorities in the management of urban sprawl have to be reordered.

In this paper I will critique the approach to the containment of urban sprawl that has dominated both the theory (academia; research) and the practice (local government; policy) in the last couple of decades and suggest four amendments to the way we see and attempt to manage the physical growth of cities. I choose to call them 'amendments' because I am conscious that not everything is wrong with the dominant paradigms in either the growth management, or the urban sustainability movements. I am not suggesting a revolution, but a correction of the course, or a shift in emphasis.

The four amendments, or corrections of the course, are:

1) less attention on transport, more on total metabolism;

2) less focus on sustainability, more on resilience; 
3) less focus on the city, more on its region;

4) less planning, more design.

After briefly tackling the wicked problem of 'urban un/sustainability' (and the associated idea of 'the sustainable city') I will attempt to present the basic argument for each of the four amendments.

\section{Sustainability of cities: metabolism, not density}

Urban sustainability' is awkward both as term (expressions) and a concept (idea). As a term, it suggests that there is, or can be, something 'urban' about sustainability. Like in the expressions we have been using for much longer: 'urban design', or 'an urban centre', or 'urban infrastructure'. Clearly, in these terms the adjective 'urban' is used to denote the urban objective or location of the nouns it accompanies; in the case of 'urban sustainability' it is really a lazy way of saying 'the sustainability of cities'.

With the idea of 'urban sustainability', it is even worse. In the broadest sense, we could define it as being able to maintain a town or a city as a livable place for humans indefinitely. History, however, teaches us that while cities tend to last over many centuries, if not millennia, there are no guaranties that they will last 'indefinitely'. Cities actually appear rather improbable, if we stop for a moment and think of all the forces stacked against them - natural disasters, fires, epidemics, wars, political unrest, etc.

In our present time, of all the various adversities preying on cities, the capacity of nature - understood as the city-supporting ecosystem - has emerged as the key issue. Will the local and regional ecosystems - and in an aggregate sense, the entire global ecosystem, or the biosphere - be able to support cities for future generations? Or, alternatively, are cities more likely to face a deep and prolonged crisis? Perhaps even an abrupt and catastrophic breakdown of the ecological balance of the entire planet - since we are relentlessly consuming resources at a rate far above the planet's ability to regenerate? Will this decline in fact a disaster of global scale - happen sometime in the latter half of this century?

The evidence is mounting that this is actually quite likely. There is a growing consensus among several authoritative and independent sources that the 'tipping point' is very close. Some even claim we have just passed the tipping point. Others say we have only 10 to 30 years to change radically the way we build and operate our cities, and among themselves, they only differ on whether they see the necessary political and technological changes coming fast enough, or not.

The daunting prospect that, in a few decades, the current rate of economic and demographic growth will almost certainly produce an ecological, and then inevitably, a global security breakdown, surely makes urban ecological sustainability the central issue of our age.

This stark prospect should, as they say, help focus the minds. But the trouble is that ecological sustainability of cities is a conundrum of sorts - it is a 'wicked problem'. It is a concept difficult to define, and it is a phenomenon difficult to measure. Indeed, there are two fundamentally opposed views on whether 'urban 
ecological sustainability' is a viable proposition at all. One could be labeled as 'pessimistic', the other as 'optimistic'. The pessimistic view suggests that 'urban sustainability' is an oxymoron. Cities cannot, and will never be sustainable; being 'nature-friendly' was never their brief. On the contrary, within the ecology of this planet, cities are irrevocably parasitic. And this will never change, at least not as a principle. What this means for averting the looming global ecological crises is that the best we can do is to hugely reduce the cities' consumption and thus bring it within the ecosystem's capacity to generate resources and assimilate waste. In other words, we may succeed in turning the 'mad' parasite' into a 'reasonable' parasite, but its fundamental inability to be productive in thermodynamic terms will stay as it is.

The other, optimistic view of urban sustainability accepts that today's cities are indeed parasites, but then goes on to argue that they nevertheless can also be viewed as a form of ecosystem. Once we adopt this view, we shift our attention onto how to redesign cities so that, similar to the natural ecosystems, they perform in some kind of a bio-productive mode. In other words, while cities historically have not been productive in neg-entropic terms, now is the time to radically change this. A huge task again, as many things will have to change simultaneously - people's perception and behavior; legislation; economic policy; planning rules; design paradigms; the market for technologies. It sure sounds big and difficult, but it is not impossible. And in any case, we do not seem to have a choice.

Therefore, the 'city-as-an-ecosystem' model is the model of hope - or perhaps even an act of faith! If we take the risk of global environmental and political meltdown as real and not distant at all, then no government has other choice but to legislate a new economic game (emissions trading; carbon tax; etc) and steer urban development into a new era.

For this new 'era of the eco-city' to actually commence, the urban ecological sustainability agenda will have to expand. Its present almost singular focus with the fossil-fuel-powered individual mobility and its calls for reducing travel distances by shrinking the city, needs to be replaced with a more comprehensive view of the manifold ways in which cities squander natural resources. Campaigns for 'urban intensification' as a way to: (i) reduce automobile dependence and reduce the need for transport overall; (ii) make investment into public transport more economic; (iii) protect habitat and landscape from development - are not without a point, but should become just another component of a wider strategy to achieve sustainable urbanization. The entire 'urban metabolism' - the overall throughput of matter, water and energy in the city - should be considered and tackled, not just fossil fuel dependency and greenhouse gas emissions.

The coming improvements in vehicle efficiency and the gradual introduction of cleaner fuels (ethanol, electricity from renewable sources, hydrogen) are likely to lessen the current concern with the automobile anyway. Also, while the desire by many households and businesses to locate outside the city proper might remain, it will be tempered by the growing costs of fuel, vehicle registration and compliance, and in some cases, road charges. 
The combined outcome of all these forces pulling in different directions is likely to be an overall moderation of the excessive individual mobility we see today, even without much planning intervention. Consequently, 'car dependency' is bound to become a lesser evil than it is today.

Whatever extra time and energy becomes available in the urban planning departments across the world as a result of relaxing attitudes towards the 'evil automobile', may then be used to tackle the other great consumer of space and resources - buildings. Buildings are responsible for more than half of the overall urban resource consumption, including enormous fossil fuel consumption, and associated emissions. The efforts of policy makers, planners, designers and engineers should be directed more at the potential for massive savings and efficiency improvements in buildings, and for dispersed, local (often buildingbased) energy generation and water harvesting. This would lead towards big changes in the way urban infrastructure is provided, as well as major cultural and behavioral shifts in urban lifestyles.

Once urban infrastructure, and the above-the-ground development it supports, become far more efficient, decentralized and localized, planners and regulators can relax their focus on 'curbing urban sprawl' and 'densifying the urban fabric' on the pretext that, at low densities, reticulated services are too expensive.

The argument for denser, traditional urban living will be still there, but this will be just another planning objective, and yet another lifestyle choice among many. The sub-urban and the peri-urban patterns of development, once transformed onto a substantially greener mode of metabolism regardless of their density, will be equally acceptable as the traditional urban.

\section{From sustainability and mitigation, to resilience and adaptation}

The international debate on climate change has recently seen an important shift in the overall discourse: more and more, scientists, international institutions and governments are talking about adaptation to climate change, not just mitigation or prevention. Obviously, much precious time was lost during the last two decades in the exhausting debates about whether climate change was happening or not, and now that we know it is happening, it is too late to prevent it. Consequently, adaptation measures are now on the agenda too, not just mitigation. This, again, has big implications for the planning and operation of cities.

An additional factor is 'peak oil'. The steadily rising price of petroleum on the world market speaks of the urgent need to consider major adaptations in the way we live in cities. As if that wasn't painful enough, mostly recently the prospect of 'peak water' (particularly underground water) has been raised. The two 'peaks' are making the whole urban situation look rather ominous from angles independent from climate change. There is a growing fear of either disruption of oil and water supplies, or, for some people, of prohibitive prices.

The dependency of cities for basic commodities - and let's not forget here food, itself heavily dependent on oil and water - from somewhere else has 
created a uneasy situation, Both city authorities and citizens are starting to wonder whether the steady, secure and cheap supply of basic necessities of life is becoming a thing of the past. The feeling of uncertainty is further reinforced by occasional local crises, like power blackouts, petrol price hikes, water rationing, food shortages, as well as the gloomy global outlook for an over-populated and highly urbanized planet.

This new situation is giving rise to what is really common sense - that we should rely more on the local supply. ('Local supply' in this case may mean several levels: from the national market, to the regional hinterland and the local neighborhood, to your own back yard and your own house roof.)

The key point here is that the already experienced, as well as the anticipated future shortages, are casting doubt on the wisdom of the centralized, 19th century model of urban infrastructure. The present model - which was created 200 years ago, in order to deal with urban squalor of the 18th century, and then enthusiastically and widely adopted as the very embodiment of 'progress' in the 20th century - is now threatening to implode under the weight of its own success. The model is based on a substantial separation of the point of production (the power plant; the water treatment plant; the gasworks) and the point of consumption (e.g. a home; office; factory; mall). Traditional urban infrastructure makes most of the city functions highly dependent on a regular supply of key resources. A state of such high dependency is by definition a state of low resilience.

As the world seems poised to enter a period of uncertainty over the availability of key resources, it is increasingly sensible to ask: how resilient are our cities to possible disruptions in the supply of such key commodities like fuel, power, water and food? While this by no means suggests we should give up the struggle to reduce the massive urban contribution to the global environmental degradation and make our presence on the planet more sustainable, it does suggest that government authorities must urgently realize and assess our cities' level of exposure to the resource challenges lying in the turbulent times ahead.

\section{Forget the city: it is the urban region now}

The dual goal of the previous argument was to broaden the urban sustainability agenda along two dimensions:

1. The range of natural resources affected by urbanization (the point here being that the total urban metabolism should be the main target of study and intervention, rather than just transport and habitat/soil destruction);

2. The nature and scale (or location) of risk (the point here being that the growing issues of resilience and adaptation must be at least equally addressed as the already adopted agenda for sustainability and mitigation).

This, hopefully, provides us with a comprehensive framework of issues that need to be examined if we are to produce an effective sustainable urbanization policy. The next question is, however, what do we mean by 'urbanization' in the 21 st century? 
To answer this, we must give appropriate consideration to the peculiar geographic characteristics of contemporary urbanization. There is plenty of evidence that the overall trend in the pattern of settlement in the last quarter of the 20th century has been one of increasing concentration of urban and infrastructure development in a small number of urban regions. At the same time, however, within these regions, the overall population density has been dropping. Households and businesses increasingly prefer cheap, open, clean, green space outside the city, to the expensive, congested, polluted, built-up space in the city. What they stand to lose in the convenience of urban locations, they apparently compensate with widely accessible personalized transport and ever more affordable telecommunication technology.

The outcome of these preferences is that an ever larger urban and semi-urban population lives in a relatively small (though growing) number of ever larger urban regions in most countries of the world.

An additional aspect of this trend is the trend towards coastal urbanization. In almost all moderate to subtropical areas of the world, urban, semi-urban, tourist and infrastructure development along the shores of oceans, seas and estuaries is gaining momentum. Thus many of the growing urban regions (as opposed to cities in the traditional sense) of the world are actually coastal urban regions.

A common characteristic of these urban regions is the widening range of densities - from very high at the CBD, to medium-high in the inner city zone, to medium-to-low in the suburbs, to low and very low in the peri-urban belt. It is difficult to imagine how this trend towards urban decentralization - even diffusion - can be stopped by any planning measures. It is even hard to imagine how it can be stopped by the rising oil (or any fuel) prices, when the residents and businesses in these outer areas have so many means of adaptation and compensation on their disposal- if they are really determined to stay where they are. It is therefore reasonable to forecast that the city (or the conurbation) of the future is actually a multi-nodal region with widely varying levels of density of development. If, for the sake of simplicity, we take that this fuzzy settled landscape consists of three major types of fabric:

- urban (high density);

- suburban (low density);

- periurban (very low density);

then we can imagine that the city region of the future will continue to grow with all three fabrics. The three landscapes will be generated roughly evenly (i.e. including significant intensification projects in the 'urban zone') via the property market, itself more or less strongly influenced by:

(1) The (rising) costs of personal and public transport.

(2) Habitat and landscape destruction levies (also rising?).

(3) The (dropping) cost, availability and convenience of telecommunications, such as telephone, television and internet, all to some degree reducing mobility

Any ideas that the rising costs of fuel (in fact all forms of energy), or planning policy, or even both, could bring cities back 'into shape' - i.e. stop and contain (not to talk of reversing!) urban sprawl - are naive and underestimate the drivers of dispersion. 


\section{From (policy) planning to (strategic) design}

The sustainable 21 st century city will not be a city. Not even a conurbation. As explained above - and whether we as planning and design professionals like it or not - it will be a sprawling entity with fuzzy edges, but with visible cores and many different levels of density. Its physical boundaries will be vague, but its ecological limits will be strict. And, while it may look somewhat random and 'formless' from a distance, it will actually consist of concentric belts and islands of differing intensities. As we become accustomed to thinking about the city as a populated region with varying degrees of density rather than a compact structure with clear limits, the design of buildings and whatever remains of the infrastructure will become the new main target of planning policy. Planning and building regulation, heavily biased towards 'green', or 'ecological', or' sustainable, or 'environmentally friendly' design, will start generating a new sub/peri/urban landscape. This landscape, both with its per-formative (ecofunctional), and its formative (visual) features, will be increasingly integrated with the natural and farming landscape. So much so, that in many areas it will be difficult to say where suburbs and industrial zones stop, and farmland and habitat reserves start.

What the exact geographic pattern of these urban regions will be is not so important. What matters is the overall process: the trend towards concentration at the macro (national) scale will be accompanied by the trend towards dispersion at the micro (regional) scale. The challenge for central government, territorial authorities and planning and design professions will be not so much how to 'curb urban sprawl' as to how to steer growth into areas within the designated urban growth regions where it will commit the least damage - ecologically and aesthetically.

Once the urban growth is directed to the areas of the least direct environmental cost, and zoned in such a way that a reasonable degree of density and public transport and facilities provision is mandated, the next step will be to ensure that the design and technological solutions support sustainable and resilient habitation.

The latter - sustainability and resilience through eco-design and ecotechnologies - are a matter of ecologically enlightened urban, landscape and architectural design (rather than planning). The new, green practice in all three disciplines - which is already being accepted as normal and mainstream by the leading professionals in the market - will have to go hand in hand with massive deployment of clean technologies in construction, energy generation, water supply, food processing, transport etc. Most of these technologies already exist. The speed of their proliferation will depend on how fast various countries shift to the new, carbon-constrained/neutral economy.

\section{Conclusion: towards urban regions designed for resilience and sustainability}

The world is facing the massive task of converting cities to a mode of functioning which will make them: 
1. Sustainable - and thus contributing to the global effort to avoid a catastrophic level of global warming;

2. Resilient - and thus adapting to the consequences of whatever level of global warming is already inevitable and whatever shortages of basic resources are coming our way.

This enormous and urgent task is more likely to succeed if we accept that cities in most of the world will never be of the Old World type - dense and compact - and that the era of physically distinct cities is over. While different countries and regions will develop their indigenous models of urbanization in accordance with their landscape, climate, culture and economic profile - some general rules will apply in most cases:

Only the core of emerging urban regions need to be compact. This core will command a relatively high density of residents and workers and will look and feel 'urban'. Quality pedestrian and public spaces, and efficient public transport, will be of great importance. The design and technology templates for such areas are already well known. It would be a waste of precious intellectual energy to further dwell on inventing again and again urban design principles and strategies for these environments. Rather, urbanists should direct their research efforts and design creativity at figuring out sustainable and resilient solutions for emerging $s u b$ - and peri-urban areas. These are the new, fertile grounds for eco-design and eco-technology innovation. By virtue of being an investment in the outer belts of an urban region, these places are far from the old-fashioned urban infrastructure and urban services. The incentives for them to be sustainable and resilient are both ecological and economic.

In fact, the room for eco-tech innovation is huge in all three belts of the contemporary urban region. The expected wave of economic incentives designed to entice clean technologies will see to that. In other words, in the coming carbon-neutral economy, the rewards for companies and nations willing to lead urban development and associated industries in this direction will be massive. But the main rewards will be at the societal level. By taking the path of clean urban development and redevelopment, we will be able to support the growing urban population without excessive cost to nature, along with a high degree of confidence in a stable supply of the key resources for life. Together, the two add up to a reasonable degree of security that our urban civilization has a future.

\section{References}

[1] Auckland Regional Council, Auckland's Sustainability Framework: www.arc.govt.nz/auckland/sustainability/auckland-sustainabilityframework.cfm

[2] Bogunovich, D (2008) Eco-Tech Urbanism: Towards the Green and Smart City. In New Urbanism and Beyond - Designing Cities for the Future. T. Haas, ed. Rizzoli, New York.

[3] Bogunovich (2008) "Eco-Tech Urbanism: Merging Urban Design with Clean Technology". To be presented at the First International Conference on Eco-Tech Cities at the Gazi University, Ankara, September 2008. 
[4] Droege, P (2006) The Renewable City - The Coming Urban Revolution. Wiley, Chichester.

[5] European Urban Charter II. Adopted at the 15th Plenary Session, 27-19 May 2008, Strasbourg. Council of Europe.

[6] McKibben, B (2006). The Coming Meltdown. In The New York Review of Books, Vol 53, No 1, January 12, 2006.

[7] Newman, P. (2008) Cities as Sustainable Ecosystems - \Principles and Practices. Island Press, Washington.

[8] State of The World 2007 - Our Urban Future. The Worldwatch Institute, Washington DC.

[9] UN's Millennium Assessment Report (http://www.millenniumassessment. org

[10] UNEP's GEO 4 - Global Environment Outlook Report: www.unep.org/ geo/geo4/media/

[11] UNEP's IETC, Cities could/should be sustainable ecosystems: www.unep.or.jp/ietc/Activities/Cross-Cutting/CASE.asp

[12] UK Govt, Stern Review 2007: www.hm-treasury.gov.uk/independent reviews/stern_review_economics_climate_change/sternreview_index.cfm

[13] Also: Jonathan Porritt, $\bar{A}$ - Gore, James Kunstler, Richard Heinberg, Jarrod Diamond and George Monbiot. 